Bond University

Research Repository

\title{
Effects of Equal Volume But Different Plyometric Jump Training Intensities on Components of Physical Fitness in Physically Active Young Males
}

Ramirez-Campillo, Rodrigo; Moran, Jason; Drury, Benjamin; Williams, Mark; Keogh, Justin W; Chaabene, Helmi; Granacher, Urs

Published in:

Journal of Strength and Conditioning Research

DOI:

10.1519/JSC.0000000000003057

Licence:

Unspecified

Link to output in Bond University research repository.

Recommended citation(APA):

Ramirez-Campillo, R., Moran, J., Drury, B., Williams, M., Keogh, J. W., Chaabene, H., \& Granacher, U. (2021).

Effects of Equal Volume But Different Plyometric Jump Training Intensities on Components of Physical Fitness in Physically Active Young Males. Journal of Strength and Conditioning Research, 35(7), 1916-1923.

https://doi.org/10.1519/JSC.0000000000003057

\section{General rights}

Copyright and moral rights for the publications made accessible in the public portal are retained by the authors and/or other copyright owners and it is a condition of accessing publications that users recognise and abide by the legal requirements associated with these rights.

For more information, or if you believe that this document breaches copyright, please contact the Bond University research repository coordinator. 


\section{EFFECTS OF EQUAL VOLUME DIFFERENT PLYOMETRIC JUMP TRAINING INTENSITIES ON COMPONENTS OF PHYSICAL FITNESS IN PHYSICALLY ACTIVE YOUNG MALES}

Rodrigo Ramirez-Campillo ${ }^{1}$, Jason Moran ${ }^{2}$, Benjamin Drury ${ }^{2}$, Mark Williams $^{2}$, Justin W. Keogh ${ }^{3,4,5}$, Helmi $^{2}$ Chaabene $^{6}$, Urs Granacher ${ }^{6}$.

${ }^{1}$ Laboratory of Human Performance. Research Nucleus in Health, Physical Activity, and Sport. Quality of Life and Wellness Research Group. Universidad de Los Lagos (University of Los Lagos). Osorno, Chile.

${ }^{2}$ Hartpury University, Hartpury, Gloucestershire, United Kingdom.

${ }^{3}$ Faculty of Health Sciences \& Medicine. Bond University. Gold Coast, Australia.

${ }^{4}$ Sports Performance Research Centre New Zealand, AUT University, Auckland, New Zealand

${ }^{5}$ Cluster for Health Improvement, Faculty of Science, Health, Education and Engineering, University of the Sunshine Coast, Australia

${ }^{6}$ Division of Training and Movement Sciences, Research Focus Cognition Sciences, University of Potsdam, Potsdam, Germany.

\section{Corresponding author}

Prof. Urs Granacher, PhD

University of Potsdam, Research Focus Cognition Sciences. Division of Training and Movement Sciences Am Neuen Palais 10, Building 12, D-14469 Potsdam (Germany)

E-mail: urs.granacher@uni-potsdam.de

Tel: +49 (0)3319771543

Fax: +49 (0)3319774022

Orcid: http://orcid.org/0000-0002-7095-813X 
EFFECTS OF EQUAL VOLUME DIFFERENT PLYOMETRIC JUMP TRAINING INTENSITIES ON COMPONENTS OF PHYSICAL FITNESS IN PHYSICALLY ACTIVE YOUNG MALES 


\section{ABSTRACT}

An 8-week single-blind randomized controlled trial was conducted to compare the effects of separate programs of equal volume, but different intensity, plyometric jump training (PJT) on physical fitness in healthy adults. Thirty-eight physically active males (mean age: 21.8 \pm 2.5 years) participated. Subjects' were randomly assigned to one of three PJT groups or a control (CON, n=9) according to their jump performance. PJT was conducted at maximal (PJT-100, $\mathrm{n}=10)$, high (PJT-80, $\mathrm{n}=9$ ) or moderate (PJT-65, $\mathrm{n}=10)$ intensity within each group. Baseline and follow-up tests were carried-out for the assessment of countermovement jump height [CMJ], CMJ height with arm swing [CMJA], and drop-jump height from a 20-cm drop-box [DJ20], linear speed (30-m), and change-of-direction speed (CODS) (Illinois-CODS test). Results revealed significant groupxtime interactions for CMJ, CMJA, DJ20, 30-m sprint, and CODS (all p<.001; $d=0.39-0.76$ ). Post-hoc analyses showed significant improvements in all five fitness measures for PJT-100 (all p<0.01, $\Delta 3.7-13.5 \%$, $d=0.26-1.4)$. For PJT-80, three out of five fitness tests demonstrated significant change (CMJ: $<<0.001, \Delta 5.9 \%$, $d=0.33$; CMJA: $\mathrm{p}<0.001, \Delta 7.0 \%, d=0.43)$; CODS: $\mathrm{p}<0.001, \Delta 3.9 \%, d=0.9)$ and for PJT-65 only 1 test was significant $(\mathrm{CMJ}: \mathrm{p}<0.05, \Delta 2.8 \%, d=0.15)$. No significant changes were observed in CON. Except for similar gains in DJ20 and 30-m sprint in PJT-100 and PJT-80, gains in physical fitness were, in general, greater ( $\mathrm{p}<0.05)$ following PJT-100 vs. PJT-80 vs. PJT-65 vs. CON. Therefore, maximal PJT intensity may induce larger physical fitness gains, although high and moderate intensities may also be useful, but to a lesser extent. KEYWORDS: plyometric exercise; stretch reflex; muscle strength; athletic performance; resistance training. 


\section{INTRODUCTION}

The stretch-shortening cycle (SSC) is a complex neurophysiological trait (38). The SSC is often targeted during training to enhance athletes' preparedness for competition. Interventions to improve SSC utilization include jump drills, commonly referred to as plyometric jump training (PJT). There is evidence that various PJT interventions (24) are effective in improving SSC function which translates to better performances in jumping, sprinting, and change-of-direction-speed (CODS) (18).

Common characteristics of PJT interventions have recently been described in a scoping review (24). To implement safe and effective PJT programs, subjects' physical fitness levels (19), together with various exercise prescription factors (e.g., type of jump drill, training frequency and volume, training surface, etc.) must be taken into account (24). Besides these factors, another key component of PJT protocols is exercise intensity (6). Previously, PJT intensity has been defined as a training-induced strain delivered to muscles, connective tissue, and joints (10). Surprisingly, no well-controlled study has examined the potential effects of different PJT intensities on components of physical fitness in athletes and/or physically active subjects. Preliminary studies attempted to identify adequate PJT intensities in both, young and elite athletes $(2,25)$. In addition, anecdotal recommendations on PJT intensities are also available in the literature (21). However, such pilot studies are either limited due to their cross-sectional design (2) or because they lack adequate control to elucidate intensity-specific dose-response relationships (25).

Furthermore, different physiological and/or biomechanical measures of PJT intensity have been applied previously (i.e., electromyographic activity $[\mathrm{EMG}]$; reactive strength index; jump height) $(2,11,12)$. These rather heterogeneous measures make it difficult to identify an adequate marker of PJT intensity. In addition, the use of some PJT intensity markers (i.e., EMG, force plates) is often restricted because expensive, laboratory-based and time-consuming apparatus are required. This prevents the wide-spread use of these 
markers. Moreover, the intensity rating of PJT may differ as a function of drill type and measuring method. For example, a drop jump can be rated as more intense compared with a box jump if ground-reaction forces or muscle-damage markers are considered $(12,41)$. However, when EMG is used as an intensity marker, the box jump is more intense $(12,41)$. In addition, controversy exists with regard to the intensity of identical drills under different conditions. For example, greater EMG activity has been shown when performing drop jumps from a $60-\mathrm{cm}$ box compared with a $20-\mathrm{cm}$ box (20). This is indicative of larger PJT intensities from more elevated drop heights. However, not all studies support these assertions (12). For example, it has also been shown that power output and reactive strength performance may augment if drop height is increased from low to medium heights. However, if drop height is further increased from medium to high, overall muscle performance is inhibited $(2,13)$. In this sense, the drop jump drill is somewhat limited as a model to study the dose-response relationship between different PJT intensities and their effects.

The inhibitory effect described above can be observed when drop jump height is greater than an individual's maximal vertical jump capacity (i.e., countermovement jump with arms [CMJA]) (2). Indeed, it has been observed in cross-sectional studies that a progressive increase from sub-maximal to maximal jump heights results in a subsequent linear increase in intensity markers (i.e., work; power; height; early onset of muscle activity) (17). However, these are findings from cross-sectional and not training intervention studies. Accordingly, this research area requires further investigation. The identification of moderate, high, and maximal exercise intensities could enable coaches to more efficiently target and design effective PJT programs. Therefore, the purpose of this study was to compare the effects of equal volume different PJT intensities (i.e., $100 \%$ [maximal], $80 \%$ [high], and 65\% [moderate] of maximal vertical jump performance) on components of physical fitness in young physically active males. According to the relevant literature (17), greater improvements in components of physical fitness were expected at maximal PJT intensities, although a significant improvement in a number of these assessments was expected after all PJT intensities. 


\section{METHODS}

\section{Experimental Approach to the Problem}

A single-blind (i.e., researchers) randomized controlled trial was conducted to compare the effects of 8 weeks of PJT with three experimental groups exercising at intensities of $100 \%$ (i.e., maximal), $80 \%$ (i.e., high) or $65 \%$ (i.e., moderate) of their maximal vertical jump performance. A control group was incorporated into the study design. Subjects exercised two times per week and performed equal PJT volumes at the described intensities. All subjects had at least 1 year of resistance training experience. Baseline and follow-up tests included the assessment of countermovement jump [CMJ], CMJ with arm swing [CMJA], and drop jump from a 20-cm drop-height [DJ20], speed (30-m linear sprint-time test), and change of direction speed (Illinois CODS test). Two weeks prior to the start of the study, two familiarization sessions were conducted which included PJT exercises and testing procedures, each lasting 30-min.

\section{Subjects}

The following inclusion criteria were considered: (i) $\geq 12$ months of systematic free-weight or machine-based resistance training, (ii) no musculoskeletal injuries for the last two months prior to the start of the study, (iii) subjects familiarized with PJT but did not conduct systematic PJT over the last 5 months, (iv) no medical problems that could compromise study participation, and (v) absence of any lower-extremity surgery during the previous two years. Subjects with a resistance training background were recruited to reduce potential musculoskeletal problems associated with repeated jumping activities. From 51 volunteers, 6 were unable to participate due to $\geq 1$ of the inclusion criteria. The remaining 45 subjects were randomly assigned to three PJT groups and a control group. After completion of the intervention, another 7 subjects had to be removed from the study because they did not follow the targeted adherence rate (i.e., had more than three absences from training) or did not present for post-training tests. Therefore, 38 physically-active young males (mean age: 
21.8 \pm 2.5 years) participated in this study. Due to a limited number of available subjects, we could not additionally include an active control group and an experimental group that followed standardized nutrition to control for potential effects of changes in physical activity behavior and nutrition. To rule out these potential sources of bias, we standardized physical activity behavior and diet over the course of the study by asking the subjects to maintain their physical activity status and diet throughout the intervention.

The three PJT groups exercised at three different training intensities: maximal (PJT-100, n=10), high (PJT-80, $\mathrm{n}=9$ ), and moderate (PJT-65, $\mathrm{n}=10$ ), based on their maximum vertical jump performance in the CMJA. Nine individuals participated in the passive control group $(\mathrm{CON})$. The randomization sequence was generated electronically (https://www.randomizer.org) and was concealed until interventions were assigned. Subjects' characteristics are displayed in Table 1. The sample size was determined using data from a previous study on the effects of PJT on vertical jump height in soccer players (29). An a priori power analysis revealed that 7 subjects per-group would be sufficient to yield $80 \%$ statistical power at the $\alpha 0.05$ level.

\section{***Table 1 here ${ }^{* * *}$}

All recruited subjects were physically active without being involved in any competitive events. Before the start of the study, subjects completed five to ten training sessions per week, each involving 30 to 40 minutes of aerobic training (i.e., moderate-intensity continuous; high-intensity interval training), 60 to 120 minutes of resistance training, and sporadic recreational sports (e.g., soccer; volleyball; basketball; cycling; athletics). Irrespective of the experimental group (including CON) considered, all subjects spent a similar amount of time per week on aerobic and resistance training. The 4 groups participating in the study usually incorporated 6-12 resistance training exercises per session (for the lower-body, upper-body and core muscles), 2-5 sets per exercise, 4-12 repetitions per set (60-90\% 1RM), with inter-set rest between 1-4 minutes. 
The study was carried out in accordance with recommendations of the latest version of the Declaration of Helsinki. The protocol was approved by the local review board from the Department of ***blind for review purposes***, University $* * *$ blind for review purposes $* * *$. Before the start of the study, subjects were informed about the experimental procedures and any possible harms and benefits. Written informed consent was obtained from all subjects before the beginning of the study.

\section{Procedures}

Before and after training, physical fitness tests were scheduled. These were carried out $\geq 72 \mathrm{~h}$ after the most recent training session to allow proper recovery from training. All tests were conducted on the same indoor gymnasium with a wooden floor, between 18:00 and 21:00 hrs. Subjects used the same sports clothes that they usually wore during training. The same experienced observers, who were blinded to group allocation, conducted all measurements. Subjects were asked to perform at their maximum effort for the tests which were carried out on a single day. Before fitness testing started, data on age, stature and body mass were recorded. Thereafter, subjects performed the physical fitness tests in the following sequence: CMJ, CMJA, DJ20, 30-m linear sprint, and Illinois CODS. Three trials were recorded for each test, and the best trial was used for further data analysis. A rest interval of at least 2-min was provided between trials. While waiting, subjects performed low-intensity activities (i.e., walking; submaximal test practice) to maintain their readiness for subsequent testing. Ten minutes of general (i.e., submaximal running with changes of direction) and specific (i.e., jumps; accelerations; kicks) warm-up exercises (1) were performed before testing started.

Anthropometry. Stature was measured using a stadiometer to the nearest $0.1 \mathrm{~cm}$ (Bodymeter 206, SECA, Hamburg, Germany). Body mass was tested using an electrical scale to the nearest $0.1 \mathrm{~kg}$ (InBody120, model BPM040S12FXX, Biospace, Inc., Seoul, Korea). 
Vertical Jump Tests. All jump tests (CMJ, CMJA, DJ20) were performed according to previous recommendations (29). Jump height was assessed to the nearest $0.1 \mathrm{~cm}$ while subjects executed maximal-effort jumps on a validated mobile contact mat (Ergojump Globus, Codogne, Italy) (22). Experienced observers ensured, through visual inspection, that take-off and landing were standardized to the relative same position on the ground. If excessive (i.e., judged by the experienced observed) forward-backward or side-to-side movements occurred during a jump, the trial was repeated after 2 minutes of rest. In addition, subjects were asked to perform full hip, knee and ankle extensions during the flight phase. For the DJ20 test, subjects were also instructed to jump for maximal height and minimal ground contact time, to maximize reactive strength index. A drop jump from a $20-\mathrm{cm}$ drop-height was used to maximize reactive strength performance and to reduce musculotendinous stress due to subjects' lack of experience of plyometric training (2). Subjects received the following instruction: "jump as high as possible and keep ground contact time minimal”. Of note, ground contact times $<250 \mathrm{~ms}$ were required for the DJ20. If ground contact times were $>250 \mathrm{~ms}$, the test trial had to be repeated.

Linear Sprint and Illinois CODS Performances. Sprint time was assessed to the nearest $0.01 \mathrm{~s}$ using singlebeam timing gates (Brower Timing System, Salt Lake City, Utah, USA). Subjects started in a standing position with the toe of the dominant foot placed behind the starting line. The test was initiated when the subject voluntarily started the sprint. The timing gates were positioned at the start line (0.3-m in front of the subjects). They were positioned $\sim 0.7 \mathrm{~m}$ above the floor (i.e., hip level), allowing us to capture trunk movement only and to avoid a false trigger from a limb. For the Illinois CODS test, the timing system and procedures were the same as for the 30-m linear sprint test except that subjects had to run as quickly as possible while performing several pre-determined changes of direction (32). 


\section{Training Program}

Plyometric jump training lasted eight weeks with two sessions per week. Previous studies indicated that this intervention period is adequate to induce significant improvements different types of physical fitness $(18,24)$. Plyometric jump training was immediately performed after the warm-up program (26). The three PJT programs replaced two aerobic training sessions per week, which amounted to $<6 \%$ of the total training load. Sporadic recreational sports were not accounted for. The CON followed its regular training program. Plyometric jump training was applied two times per week, with a $48 \mathrm{~h}$ rest between-sessions. The PJT protocol was based on previous research findings (17), with the main drill used during training being the CMJA. This bilateral and acyclical (i.e., non-repetitive) drill affords a maximal vertical jump that involves a slow SSC movement $(\geq 250$ ms). For all PJT groups, a strong emphasis was put on landing technique and shock absorption, using medium to hard (i.e., 3-cm thick mat) and hard (i.e., wood floor) training surfaces (14).

Subjects in the PJT-100 group were asked to jump at maximal effort in a vertical direction for each jump. Thus, they were supposed to achieve the same, or better, performances compared with the best pre-intervention CMJA trial. To ensure that subjects completed each jump at $100 \%$ of their maximum vertical jumping capacity, a mark was positioned on a nearby wall and used as an external cue. Another mark was positioned at $90 \%$ of maximum CMJA height. To ensure that subjects achieved the desired intensity (i.e., jump height) during each jump, a coach-to-subject ratio of 1:1 was used during all training sessions (31). The coach supervised each jump and was attentive to the intensity and technical skill level of the jump. Notably, if subjects were unable to reach $100 \%$ of their maximum performance, they were encouraged to reach $90 \%$ of that level. A rest period of 15 seconds was granted between repetitions (34), and 120 seconds was given between sets (27). To ensure that all scheduled repetitions were completed at an intensity $\geq 90 \%$ of maximum vertical jump performance, an add libitum rest ( 1-2 minutes) was allowed before the next jump trial. For PJT-80 and PJT-65, similar approaches were followed as that described for PJT-100. Accordingly, those groups had to achieve between 
70 and $80 \%$ of their maximum jump height (PJT-80) or between 55 and $65 \%$ of their jump height (PJT-65). Again, a vertical mark was positioned at $80 \%$ and $65 \%$ of maximum pre-intervention CMJA performance. All subjects in the PJT-80 and PJT-65 were at least able to train at 70-80\% and 55-65\% of their maximum vertical jump performance throughout the 8 weeks of the PJT program. Maximal CMJA performance was reassessed after the first four weeks of training, with this new CMJ peak performance used as the subjects' jump height target for the final four weeks of the intervention.

The PJT program is described in detail in Table 2. Each PJT session included 10 sets of CMJA drills, with 410 repetitions per set. As previously suggested (30), the volume of training was increased progressively from the $1^{\text {st }}$ ( 4 repetitions per set), toward the $7^{\text {th }}$ week of training (10 repetitions per set). During the $8^{\text {th }}$ week, a taper of training volume was applied (5 repetitions per set). All PJT sessions lasted 30-45-minutes and all intervention groups performed a total of 1080 jumps during the program. Identical weekly progressive overload and volume-based taper strategies were performed for all training groups. In addition, all PJT groups trained

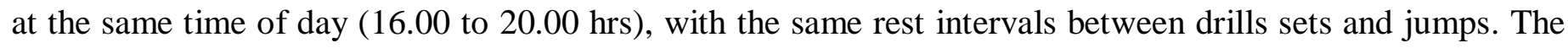
only between-group difference was the intensity of the vertical jumps.

***Table 2 near here***

\section{Statistical Analyses}

Data are presented as group mean values and standard deviations. After data normality was verified with the Shapiro-Wilk test, a one-way analyses of variance (ANOVA) was used to detect baseline between-group differences and percentage rates of pre-to-post-training changes. A two-way ANOVA with repeated measures 
on time was used to group-specifically analyze all dependent variables (Groups: PJT-100, PJT-80, PJT-65, $\mathrm{CON}) \times 2$ (Time: pre, post). Post-hoc tests with Bonferroni adjustment were conducted to identify comparisons that were statistically significant. Effect sizes for main effects of 'group' and ‘time' as well as Group×Time interactions were taken from the ANOVA output (partial eta squared). Within-group Cohen's $d$ effect sizes were computed (8), using the following equation: effect sizes $=$ (mean_post - mean_pre)/pooled standard deviation. A detectable effect size $(d)$ of $0.2(15,23)$ was considered as meaningful. Statistical analyses were carried out using STATISTICA statistical package (Version 8.0; StatSoft, Inc., Tulsa, USA). Significance levels were set at $\alpha=5 \%$. The reliability of all dependent variables was acceptable (16), with intra-class correlation coefficients values amounted to $0.97,0.95,0.93,0.91$ and 0.89 for the CMJ, CMJA, DJ20, 30-m sprint, and CODS tests, respectively, and coefficients of variation $<4.2 \%$.

\section{RESULTS}

From the subjects considered for data analyses, all received the treatment as allocated and all groups reported no changes in their training habits during the 8 weeks of the intervention, as compared to their training routine prior to recruitment. Additionally, adherence rates were similar for the three PJT groups with a mean of $15.5 \pm 0.7$ training sessions $(96.8 \pm 4.3 \%$ of all training sessions $)$.

No significant between-group baseline differences were observed for any fitness or descriptive measurements (Table 3). The main effects of group, time, and group $\times$ time interactions are displayed in Table 3.

***Table 3 near here ${ }^{* * *}$ 
Results revealed significant main effects of time for CMJ, CMJA, DJ20, and CODS (all $\mathrm{p}<0.001 ; d=0.26-0.79$ )

(Table 3). Additionally, significant group $\times$ time interaction effects were observed for CMJ, CMJA, DJ20, 30m sprint, and CODS (all p<0.001; $d=0.39-0.76)$ (Table 3).

Post-hoc analyses revealed significant improvements for all 5 physical fitness measures for PJT-100 (CMJ: $\mathrm{p}<0.001, \Delta 9.3 \%, d=0.45 ;$ CMJA: $\mathrm{p}<0.01, \Delta 13.5 \%, d=0.57 ; \mathrm{DJ} 20: \mathrm{p}<0.01, \Delta 7.2 \%, d=0.26 ; 30-\mathrm{m}$ sprint: $\mathrm{p}<0.001, \Delta 3.7 \%, d=0.73 ;$ CODS: $\mathrm{p}<0.001, \Delta 8.2 \%, d=1.4)$. For PJT-80, post-hoc analyses demonstrated significant increases in three out of five physical fitness measures, namely CMJ ( $<<0.001, \Delta 5.9 \%, d=0.33)$, CMJA $(\mathrm{p}<0.001, \Delta 7.0 \%, d=0.43)$, and CODS $(\mathrm{p}<0.001, \Delta 3.9 \%, d=0.9)$. For PJT-65, post-hoc results demonstrated significant increases in only one test (CMJ: $\mathrm{p}<0.05, \Delta 2.8 \%, d=0.15)$. No significant changes were observed for CON ( $\mathrm{p}>0.05)$. Except for similar gains in DJ20 and 30-m sprint in the PJT-100 and PJT80 groups, gains in the aforementioned physical fitness components were, in general, greater $(\mathrm{p}<0.05)$ following PJT-100 vs. PJT-80 vs. PJT-65 vs. CON.

\section{DISCUSSION}

The aim of this study was to compare the effects of equal volumes of different PJT intensities on different components of physical fitness in young physically active males. The main findings showed that greater performance improvements were associated with maximal PJT intensity. Compared with CON, all PJT groups significantly improved their jumping, sprinting, or CODS performances. Of note, gains in these physical fitness components were, in general, greater following PJT-100 vs. PJT-80 vs. PJT-65 vs. CON.

If controlled for training volume, greater levels of PJT intensity seem to result in proportionately larger increases in jump performance. Previous meta-analyses $(9,36,37)$ have highlighted the importance of PJT intensity to improve strength, speed and jumping. A recent study also supports the current findings (25) with 
male youth soccer players, trained with maximal PJT intensity for 8 weeks, using optimal drop box heights as a tool for training prescription. The applied method resulted in maximal reactive strength index development (25), underlining the importance of intensity when programming for PJT. The players who trained with maximal intensity achieved greater physical fitness improvements, compared to players who used sub-optimal drop box heights (i.e., lower intensity) (25). The current findings strengthen previous results, describing a novel dose-response relationship between PJT intensity and physical fitness improvements. However, it must be acknowledged that other studies that have utilized alternative ways to quantify PJT intensity have found somewhat different results. For example, Thomas et al. (39) compared the effects of two different PJT programs on measures of jump and agility performance in male youth soccer players. While the first PJT group performed CMJs, the second group utilized DJs, the latter drill being considered more intense that the former (12). In contrast to the findings of the present study, and a previous study in youth soccer players (25), Thomas et al. observed similar outcomes in jump height and agility performance for both groups following 6 weeks of PJT training (39).

Future studies should examine the effects of different levels of PJT intensity within and between exercises to establish a more complete overview of the relationship between intensity and the magnitude of adaptation. Special caution should also be observed at greater training loads due to the potential appearance of overreaching and/or overtraining $(7,35)$. Our current experimental approach could be further implemented by modulating training intensity through the inclusion of additional loads and/or by implementing DJ drills instead of CMJA. In particular, the use of external loads would allow the strength and conditioning coach to establish specific dose-response relationships for PJT intensity because that variable could be gradually increased. It should, however, be acknowledged that such an approach is only feasible in well-trained athletes with adequate lower body strength and power, as well as technical skill levels in jumping and landing. Further studies may also analyze the potential effects of a periodization approach in sports with specific plyometric demands (e.g., 
handball, basketball, soccer, volleyball), considering individual variations within a macro-cycle to account for athletes' fitness and fatigue levels. Additionally, whilst the results of this study demonstrate that PJT intensity in the vertical plane improves multiple components of physical fitness, it is noteworthy to consider whether such an approach would also be effective with horizontal jump training. Previously, it has been shown that a combination of vertical and horizontal PJT protocols resulted in larger fitness improvements compared with single-mode vertical or horizontal PJT (28). In other words, it is still open for debate whether training intensity for horizontal PJT has a similar impact on training-induced outcomes when compared to vertical PJT.

PJT can improve sprinting and CODS due to better SSC functioning which leads to improved movement economy (5). Supporting this finding, in their meta-analysis, Asadi et al. (4), observed PJT to be effective in improving CODS, with this improvement possibly related to improved force reutilisation through decreased ground time and increased force outputs (42). As for the current study, it is speculative to discuss potential mechanisms responsible for the larger performance improvements in PJT-100 compared with PJT-80 or PJT65. Therefore, future studies are needed to elucidate the potential neuromuscular and biomechanical mechanisms (e.g., propulsive force; motor unit recruitment; joint power) responsible for intensity-specific improvements in PJT (41). More specifically, the use of inverse dynamics could help to disentangle specific moments in the ankle, knee and hip joints during jumping at different intensities. This research question appears to be of high relevance for lower limb rehabilitation and return to play after an injury. Moreover, the potential role of landing volumes after jumping at different intensities may have potentially affected musculotendinous stiffness through different braking forces at touch down (11), contributing to increased drop jump ability. In this sense, it would be of relevance to consider if, for example, a greater PJT volume at a lower intensity would induce similar adaptations compared with lower volume and larger intensity. Moreover, an individual's level of technical competency can influence adaptive responses (40), but since all individuals started with the same level of experience in PJT, this potential source of bias can be ruled out. In addition, the observed improvements 
in the other physical fitness tests (e.g., CODS, speed) suggest that training-induced adaptations translated to different physical qualities.

Other remaining issues relate to where the findings sit with respect to the wider context of PJT and what we can infer with respect to alternative jumping formats such as drop jumping. A commentary on this was recently presented in a PJT scoping review (24), and further effort should be directed toward closing gaps related to PJT's key methodological aspects, such as training intensity. Additionally, future studies could clarify if the current results were affected by the augmented feedback provided to the athletes during training (i.e., the mark used as an external cue during jumps) $(3,33)$. Moreover, although all groups of subjects spent a similar amount of time per week on aerobic and resistance training, we did not control for the regular daily training load of the subjects. Future studies should strive to replicate the current findings by directly controlling the resistance training load and nutrient intake of the included subjects. In addition, neuromechanical and physiological assessments should be considered to better understand the underlying mechanisms of PJT induced adaptations under different training intensities. In this sense, it would be of relevance to elucidate if current findings can be replicated not only when an equal volume of jumps is completed in groups under different plyometric jump training intensities based on maximal vertical jump height, but also when other intensity-related factors are controlled (e.g., total mechanical work; electromyographic activity).

In conclusion, compared with CON, all PJT groups significantly improved measures of jumping, speed, or CODS. Of note, the PJT-100 group realized the largest performance gains compared with the other groups. Practitioners may use maximal PJT intensity to maximize physical fitness provided that the technical competency level of their athletes is sufficiently developed. High and moderate intensities proved to be effective as well. 


\section{PRACTICAL APPLICATIONS}

This study provides information for practitioners on the differences in training related responses to different intensities of PJT training, which may provide some insight into how to vary this variable over time. Although a PJT program at maximal intensity may be implemented safely (as demonstrated in the present study and in many others) from the beginning of a training plan, the use of submaximal PJT intensities may be introduced first, especially to reduce the physiological stress associated with maximal voluntary effort. Similarly, a submaximal approach may be appropriate when working with subjects who have little experience of PJT, a low level of strength, poor technical competency while jumping, or when rehabilitating an injury.

\section{REFERENCES}

1. Andrade DC, Henriquez-Olguin C, Beltran AR, Ramirez MA, Labarca C, Cornejo M, Alvarez C, and Ramirez-Campillo R. Effects of general, specific and combined warm-up on explosive muscular performance. Biol Sport 32: 123-128, 2015.

2. Andrade DC, Manzo O, Beltrán AR, Alvares C, Del Río R, Toledo C, Moran J, and Ramirez-Campillo R. Kinematic and neuromuscular measures of intensity during plyometric jumps. Journal of Strength and Conditioning Research, IN PRESS.

3. Argus CK, Gill ND, Keogh JW, and Hopkins WG. Acute effects of verbal feedback on upper-body performance in elite athletes. J Strength Cond Res 25: 3282-3287, 2011.

4. Asadi A, Arazi H, Young WB, and Saez de Villarreal E. The effects of plyometric training on changeof-direction ability: A meta-analysis. Int J Sports Physiol Perform 11: 563-573, 2016.

5. Balsalobre-Fernandez C, Santos-Concejero J, and Grivas GV. Effects of strength training on running economy in highly trained runners: A systematic review with meta-analysis of controlled trials. $J$ Strength Cond Res 30: 2361-2368, 2016. 
6. Behm DG, Young JD, Whitten JHD, Reid JC, Quigley PJ, Low J, Li Y, Lima CD, Hodgson DD, Chaouachi A, Prieske O, and Granacher U. Effectiveness of traditional strength vs. power training on muscle strength, power and speed with youth: a systematic review and meta-analysis. Frontiers in physiology 8, 2017.

7. Brumitt J, Wilson V, Ellis N, Petersen J, Zita CJ, and Reyes J. Preseason lower extremity functional test scores are not associated with lower quadrant injury - A validation study with normative data on 395 division III athletes. Int J Sports Phys Ther 13: 410-421, 2018.

8. Cohen J. Statistical Power Analysis for the Behavioral Sciences. Hillsdale, N.J. : Lawrence Erlbaum, 1988.

9. de Villarreal ES, Kellis E, Kraemer WJ, and Izquierdo M. Determining variables of plyometric training for improving vertical jump height performance: a meta-analysis. J Strength Cond Res 23: 495-506, 2009.

10. Ebben WP. Practical guidelines for plyometric intensity. NSCA's Performance Training Journal 6: $12-$ $16,2007$.

11. Ebben WP, Fauth ML, Garceau LR, and Petushek EJ. Kinetic quantification of plyometric exercise intensity. J Strength Cond Res 25: 3288-3298, 2011.

12. Ebben WP, Simenz C, and Jensen RL. Evaluation of plyometric intensity using electromyography. $J$ Strength Cond Res 22: 861-868, 2008.

13. Flanagan EP and Comyns TM. The use of contact time and the reactive strength index to optimize fast stretch-shortening cycle training. Strength \& Conditioning Journal 30: 32-38 2008.

14. Granacher U, Prieske O, Majewski M, Busch D, and Muehlbauer T. The role of instability with plyometric training in sub-elite adolescent soccer players. Int J Sports Med 36: 386-394, 2015.

15. Hopkins W. How to interpret changes in an athletic performance test. Sportsci 8: 1-7, 2004.

16. Hopkins WG. Measures of reliability in sports medicine and science. Sports Med 30: 1-15, 2000. 
17. Lees A, Vanrenterghem J, and De Clercq D. The maximal and submaximal vertical jump: implications for strength and conditioning. J Strength Cond Res 18: 787-791, 2004.

18. Markovic G and Mikulic P. Neuro-musculoskeletal and performance adaptations to lower-extremity plyometric training. Sports Med 40: 859-895, 2010.

19. Moran J, Clark CCT, Ramirez-Campillo R, Davies MJ, and Drury B. A meta-analysis of plyometric training in female youth: its efficacy and shortcomings in the literature. J Strength Cond Res, IN PRESS.

20. Peng HT, Kernozek TW, and Song CY. Quadricep and hamstring activation during drop jumps with changes in drop height. Phys Ther Sport 12: 127-132, 2011.

21. Piper TJ and Erdmann LD. A 4-Step Plyometric Program. Strength \& Conditioning Journal 20: 72-73, 1998.

22. Pueo B, Lipinska P, Jimenez-Olmedo JM, Zmijewski P, and Hopkins WG. Accuracy of Jump-Mat Systems for Measuring Jump Height. Int J Sports Physiol Perform 12: 959-963, 2017.

23. Radnor JM, Lloyd RS, and Oliver JL. Individual response to different forms of resistance training in school-aged boys. J Strength Cond Res 31: 787-797, 2017.

24. Ramirez-Campillo R, Alvarez C, Garcia-Hermoso A, Ramirez-Velez R, Gentil P, Asadi A, Chaabene H, Moran J, Meylan C, Garcia-de-Alcaraz A, Sanchez-Sanchez J, Nakamura FY, Granacher U, Kraemer W, and Izquierdo M. Methodological characteristics and future directions for plyometric jump training research: A scoping review. Sports Med 48: 1059-1081, 2018.

25. Ramirez-Campillo R, Alvarez C, García-Pinillos F, Sanchez-Sanchez J, Yanci J, Castillo D, Loturco I, Chaabene H, Moran J, and Izquierdo M. Optimal reactive strength index: is it an accurate variable to optimize plyometric training effects on measures of physical fitness in young soccer players? J Strength Cond Res 32: 885-893, 2018. 
26. Ramirez-Campillo R, Alvarez C, Gentil P, Loturco I, Sanchez-Sanchez J, Izquierdo M, Moran J, Nakamura FY, Chaabene H, and Granacher U. Sequencing effects of plyometric training applied before or after regular soccer training on measures of physical fitness in young players. J Strength Cond Res, IN PRESS.

27. Ramirez-Campillo R, Andrade DC, Alvarez C, Henriquez-Olguin C, Martinez C, Baez-Sanmartin E, Silva-Urra J, Burgos C, and Izquierdo M. The effects of interset rest on adaptation to 7 weeks of explosive training in young soccer players. J Sports Sci Med 13: 287-296, 2014.

28. Ramirez-Campillo R, Gallardo F, Henriquez-Olguin C, Meylan CM, Martinez C, Alvarez C, Caniuqueo A, Cadore EL, and Izquierdo M. Effect of vertical, horizontal, and combined plyometric training on explosive, balance, and endurance performance of young soccer players. J Strength Cond Res 29: 1784$1795,2015$.

29. Ramirez-Campillo R, Garcia-Pinillos F, Garcia-Ramos A, Yanci J, Gentil P, Chaabene H, and Granacher U. Effects of different plyometric training frequencies on components of physical fitness in amateur female soccer players. Frontiers in physiology 9: 934, 2018.

30. Ramirez-Campillo R, Henriquez-Olguin C, Burgos C, Andrade DC, Zapata D, Martinez C, Alvarez C, Baez EI, Castro-Sepulveda M, Penailillo L, and Izquierdo M. Effect of progressive volume-based overload during plyometric training on explosive and endurance performance in young soccer players. J Strength Cond Res 29: 1884-1893, 2015.

31. Ramirez-Campillo R, Martinez C, de La Fuente CI, Cadore EL, Marques MC, Nakamura FY, Loturco I, Caniuqueo A, Canas R, and Izquierdo M. High-Speed Resistance Training in Older Women: The Role of Supervision. J Aging Phys Act 25: 1-9, 2017.

32. Ramirez-Campillo R, Meylan C, Alvarez C, Henriquez-Olguin C, Martinez C, Canas-Jamett R, Andrade DC, and Izquierdo M. Effects of in-season low-volume high-intensity plyometric training on explosive actions and endurance of young soccer players. J Strength Cond Res 28: 1335-1342, 2014. 
33. Randell AD, Cronin JB, Keogh JW, Gill ND, and Pedersen MC. Effect of instantaneous performance feedback during 6 weeks of velocity-based resistance training on sport-specific performance tests. $J$ Strength Cond Res 25: 87-93, 2011.

34. Read MM and Cisar C. The influence of varied rest interval lengths on depth jump performance. $J$ Strength Cond Res 15: 279-283, 2001.

35. Ronnestad BR, Kvamme NH, Sunde A, and Raastad T. Short-term effects of strength and plyometric training on sprint and jump performance in professional soccer players. J Strength Cond Res 22: 773$780,2008$.

36. Saez-Saez de Villarreal E, Requena B, and Newton RU. Does plyometric training improve strength performance? A meta-analysis. J Sci Med Sport 13: 513-522, 2010.

37. Saez de Villarreal E, Requena B, and Cronin JB. The effects of plyometric training on sprint performance: a meta-analysis. J Strength Cond Res 26: 575-584, 2012.

38. Taube W, Leukel C, and Gollhofer A. How neurons make us jump: the neural control of stretchshortening cycle movements. Exerc Sport Sci Rev 40: 106-115, 2012.

39. Thomas K, French D, and Hayes PR. The effect of two plyometric training techniques on muscular power and agility in youth soccer players. $J$ Strength Cond Res 23: 332-335, 2009.

40. Trecroci A, Cavaggioni L, Caccia R, and Alberti G. Jump rope training: Balance and motor coordination in preadolescent soccer players. J Sports Sci Med 14: 792-798, 2015.

41. Van Lieshout KG, Anderson JG, Shelburne KB, and Davidson BS. Intensity rankings of plyometric exercises using joint power absorption. Clin Biomech (Bristol, Avon) 29: 918-922, 2014.

42. Young W and Farrow D. A review of agility: Practical applications for strength and conditioning. Strength Cond J 28: 24-29, 2006. 
Table 1. Descriptive data of the control group and plyometric jump training groups.

\begin{tabular}{lcccc}
\hline & Control $(\mathrm{n}=9)$ & PJT-65 $(\mathrm{n}=10)$ & PJT-80 $(\mathrm{n}=9)$ & PJT-100 $(\mathrm{n}=10)$ \\
\hline Age $(\mathrm{y})$ & $21.0 \pm 2.3$ & $21.8 \pm 3.1$ & $22.4 \pm 2.1$ & $22.0 \pm 2.4$ \\
Body mass $(\mathrm{kg})$ & $76.1 \pm 10.2$ & $76.0 \pm 9.8$ & $80.1 \pm 17.2$ & $79.6 \pm 17.0$ \\
Height $(\mathrm{cm})$ & $176.5 \pm 8.5$ & $176.4 \pm 4.1$ & $176.6 \pm 6.3$ & $173.9 \pm 9.7$ \\
Body mass index $\left(\mathrm{kg} \cdot \mathrm{m}^{-2}\right)$ & $24.4 \pm 1.7$ & $24.4 \pm 2.3$ & $25.5 \pm 4.4$ & $26.0 \pm 2.9$ \\
& & & \\
\hline PJT-65, PJT-80, and PJT-100: plyometric jump training using 65\%, $80 \%$ and 100\% of the maximal \\
vertical jump height intensity.
\end{tabular}


Table 2. Plyometric jump training program.

\begin{tabular}{lcccccccc}
\hline & Week 1 & Week 2 & Week 3 & Week 4 & Week 5 & Week 6 & Week 7 & Week 8 \\
& & & & & & & & \\
CMJA* $^{10 \times 4}$ & $10 \times 5$ & $10 \times 6$ & $10 \times 7$ & $10 \times 8$ & $10 \times 9$ & $10 \times 10$ & $10 \times 5$
\end{tabular}

CMJA: countermovement jump with arm swing. *: values are expressed as sets $\times$ repetitions. 
Table 3. Group-specific means and standard deviations (SD) for all outcome measures before (Pre) and after (Post) the intervention period.

\begin{tabular}{|c|c|c|c|c|c|}
\hline & \multirow[b]{2}{*}{ Pre } & \multirow[b]{2}{*}{ Post } & \multicolumn{3}{|c|}{ ANOVA outcomes } \\
\hline & & & $\begin{array}{c}\text { Group } \\
\mathrm{F}(3,34), \mathrm{p} \text {-value }(d)\end{array}$ & $\begin{array}{c}\text { Time } \\
\mathrm{F}(1,34), \mathrm{p} \text {-value }(d)\end{array}$ & $\begin{array}{c}\text { Group x Time } \\
\mathrm{F}(3,34), \mathrm{p} \text {-value }(d)\end{array}$ \\
\hline Countermovement jump (cm) & & & $\mathrm{F}=1.2, \mathrm{p}=0.33(0.1)$ & $\mathrm{F}=127.1, \mathrm{p}<0.001(0.79)$ & $\mathrm{F}=22.2, \mathrm{p}<0.001(0.66)$ \\
\hline Control $(n=9)$ & $32.9 \pm 4.9$ & $32.9 \pm 4.4$ & & & \\
\hline PJT-65 $(n=10)$ & $36.5 \pm 7.0$ & $37.5 \pm 7.1^{\mathrm{c}}$ & & & \\
\hline PJT-80 (n=9) & $35.3 \pm 6.3$ & $37.3 \pm 6.1^{\mathrm{a}}$ & & & \\
\hline PJT-100 (n=10) & $31.5 \pm 6.3$ & $34.4 \pm 6.8^{\mathrm{a}}$ & & & \\
\hline Countermovement jump with arms (cm) & & & $\mathrm{F}=0.2, \mathrm{p}=0.91(0.02)$ & $\mathrm{F}=64.1, \mathrm{p}<0.001(0.65)$ & $\mathrm{F}=12.3, \mathrm{p}<0.001(0.52)$ \\
\hline Control $(n=9)$ & $39.6 \pm 6.7$ & $40.1 \pm 7.6$ & & & \\
\hline PJT-65 (n=10) & $40.5 \pm 7.8$ & $41.6 \pm 7.8$ & & & \\
\hline PJT-80 (n=9) & $41.2 \pm 6.6$ & $44.0 \pm 6.5^{\mathrm{a}}$ & & & \\
\hline PJT-100 (n=10) & $38.4 \pm 8.4$ & $43.6 \pm 9.9^{\mathrm{b}}$ & & & \\
\hline $20-\mathrm{cm}$ reactive strength index $(\mathrm{mm} / \mathrm{ms})^{*}$ & & & $\mathrm{~F}=0.8, \mathrm{p}=0.51(0.07)$ & $\mathrm{F}=11.9, \mathrm{p}<0.01(0.26)$ & $\mathrm{F}=7.4, \mathrm{p}<0.001(0.39)$ \\
\hline Control $(n=9)$ & $1.28 \pm 0.3$ & $1.24 \pm 0.3$ & & & \\
\hline PJT-65 $(n=10)$ & $1.43 \pm 0.4$ & $1.45 \pm 0.4$ & & & \\
\hline PJT-80 (n=9) & $1.48 \pm 0.3$ & $1.55 \pm 0.3$ & & & \\
\hline PJT-100 (n=10) & $1.42 \pm 0.4$ & $1.53 \pm 0.4^{\mathrm{b}}$ & & & \\
\hline 30-m sprint time (s) & & & $\mathrm{F}=1.1, \mathrm{p}=0.36(0.09)$ & $\mathrm{F}=0.32, \mathrm{p}=0.58(0.01)$ & $\mathrm{F}=7.5, \mathrm{p}<0.001(0.40)$ \\
\hline Control $(n=9)$ & $5.14 \pm 0.2$ & $5.32 \pm 0.3$ & & & \\
\hline PJT-65 (n=10) & $5.26 \pm 0.4$ & $5.25 \pm 0.4$ & & & \\
\hline PJT-80 (n=9) & $5.09 \pm 0.2$ & $5.05 \pm 0.2$ & & & \\
\hline PJT-100 $(n=10)$ & $5.19 \pm 0.3$ & $5.0 \pm 0.2^{\mathrm{a}}$ & & & \\
\hline Change of direction speed (s) & & & $\mathrm{F}=1.1, \mathrm{p}=0.37(0.09)$ & $\mathrm{F}=59.5, \mathrm{p}<0.001(0.64)$ & $\mathrm{F}=35.8, \mathrm{p}<0.001(0.76)$ \\
\hline Control (n=9) & $18.4 \pm 1.0$ & $18.7 \pm 1.3$ & & & \\
\hline PJT-65 (n=10) & $18.4 \pm 1.1$ & $18.2 \pm 1.1$ & & & \\
\hline PJT-80 (n=9) & $18.7 \pm 0.7$ & $18.0 \pm 0.7^{\mathrm{a}}$ & & & \\
\hline PJT-100 $(n=10)$ & $18.6 \pm 0.9$ & $17.1 \pm 0.6^{\mathrm{a}}$ & & & \\
\hline
\end{tabular}


